Dawei Jia*, Huiji Shi and Lei Cheng

\title{
Multiscale thermomechanical modeling of short fiber-reinforced composites
}

DOI 10.1515/secm-2015-0487

Received November 27, 2015; accepted March 2, 2016; previously published online April 26, 2016

\begin{abstract}
A study of the micromechanical behavior to predict the overall response of short fiber-reinforced composites under cyclic mechanical and thermal loading is presented. The instantaneous average over a "representative volume" of the material is considered. The influence of the short fiber's aspect ratio, volume fraction, and spatial orientation has been investigated. The linear combined hardening model is used to describe the cyclic hardening effects in the case of metal matrix. A numerical procedure is used to predict the response of composites under mechanical and thermal conditions. The results of the numerical procedure have been compared to the results of three different models and to published experimental data.
\end{abstract}

Keywords: cyclic mechanical and thermal loading; instantaneous average; linear combined hardening model; representative volume.

\section{Introduction}

The use of fiber-reinforced materials has grown rapidly in the recent years in many applications, including aircraft, yachts, sporting goods, and motor vehicles, beside a wide range of military applications. Fiber-reinforced materials are a broad and versatile class of materials, which include a wide range of fiber and matrix combinations that provide a large number of component design and manufacturing options. High strength-to-weight ratio, coupled with excellent durability, corrosion resistance, and low maintenance cost, is among the factors that make fiber-reinforced materials ideal for widespread applications.

*Corresponding author: Dawei Jia, Engineering Research and Development Center, AVIC SAC Commercial Aircraft Company Ltd, 89 Chuangxin Road, Hunnan District, Shenyang, 110079, China, e-mail: jia.dawei@sacc.com.cn

Huiji Shi and Lei Cheng: School of Aerospace Engineering, Tsinghua University, Beijing 100084, China
Short fiber-reinforced composites enjoy the advantage of providing stiffness levels comparable to continuous fibers while at the same time being moldable into complex shapes. They are attractive in relatively low fabrication costs and constitute a major portion of the demand of composites in automotive, marine, and aeronautic applications. Figure 1 includes the composite window frame designed by the Boeing aircraft company [1].

The particular benefits exhibited by metal matrix composites, such as increased specific strength and stiffness, improved high-temperature performance limits, and better wear-abrasion resistance, are dependent on the properties of the matrix alloy and of the reinforcing phase. A variety of metal matrix composite materials are used in automotive, space systems and satellites, medical, power semiconductors, sporting goods, and several military applications. Among the multiple uses are the space shuttle frames, Hubble telescope, bicycle frames, automotive pistons, brakes, and disks.

The properties of materials depend in a complex manner on the microstructure. The strength of a fiberreinforced composite, for example, is determined by fracture criteria, themselves governed by a combination of microscopic phenomena. These include the plastic deformation of the matrix, the strength of the interface, and the distribution of the reinforcement within the composite. Therefore, predicting the strength of the composite from that of its constituent phases is generally challenging.

In the aviation industry, airworthiness specifications require an evaluation of the principal structural elements under cyclic mechanical and thermal loading as expected in service. The thermomechanical behavior of short fiberreinforced composites has been investigated at high working temperature [2,3]. However, the investigation was limited to varying mechanical loading at uniform temperature or constant mechanical loading under temperature time history. In addition, particle-reinforced composites $[4,5]$ have been studied under uniaxial tensile loading. However, these investigations were limited to mechanical uniaxial loading. In practical applications, short fiberreinforced composites are subjected to cyclic mechanical loading coupled by simultaneous varying temperatures. Furthermore, it is difficult to align the short fibers during processing in a typical production environment. 


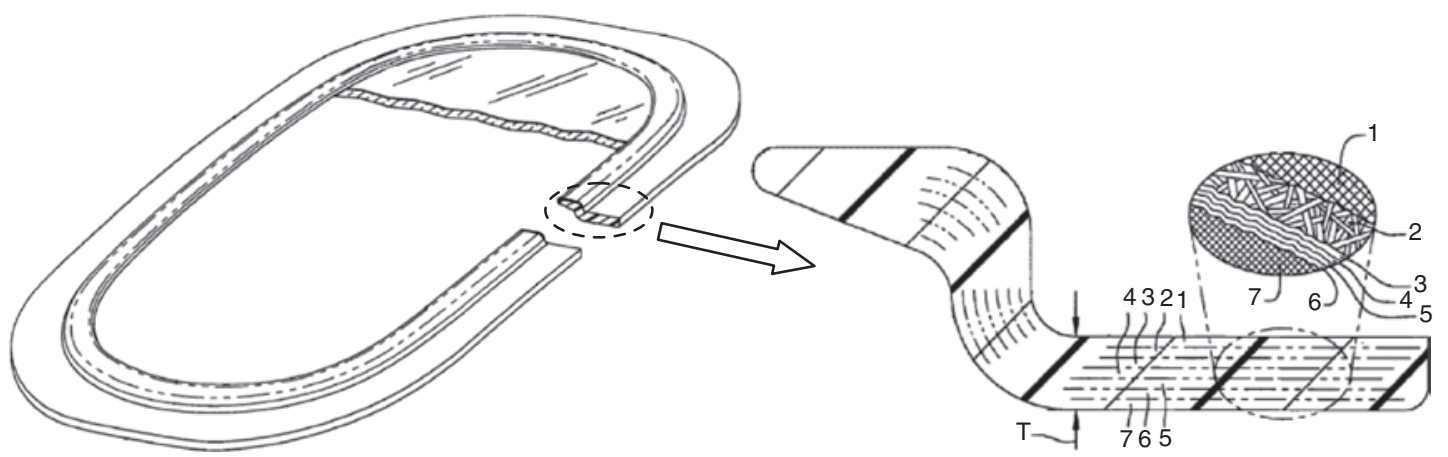

Figure 1: Composite window frame.

Cavalcante et al. [6] modeled heterogeneous materials with macroscopically uniform microstructures using either the concepts of statistical homogeneity based on "representative volume" element or periodicity based on repeating unit cell. This research demonstrated that a micromechanics model plays a key role in understanding how the local properties of constituent phases and their arrangement influence the macroscopic structural behavior. The purpose of this paper is to predict the overall response of short fiber-reinforced composites under cyclic mechanical and thermal loading and to investigate the influence of the short fiber's aspect ratio, volume fraction, and spatial orientation.

\section{Thermomechanical constitutive relations}

\subsection{Representative volume}

A representative volume element is the smallest possible volume that contains the necessary microstructure details such that the response of these elements under appropriate boundary conditions is identical to that of the material at large. The representative volume consists of matrix and short fiber phases and whose fibers are uniformly distributed, well bonded with the matrix, and identical in material and shape. A representative volume is not in a neighborhood close to the boundary. It contains sufficiently large number of short fibers to reflect the microstructure of materials yet is small enough compared to the macroscopic scale. The representative volume is selected to embody the overall response of the structure [6-8]. The shape of the short fibers is assumed to be ellipsoid, and the particulatereinforced composite is one of its particular cases.

Throughout this paper, scalars and integers are represented by italic letters, vectors and second-order tensors by lower-case boldface letters, and fourth-order tensors by upper-case boldface letters. To describe the orientation of short fibers, two coordinate systems were defined. A global coordinate system $\left(x_{1}, x_{2}, x_{3}\right)$ and a local coordinate system $\left(x_{1}^{\prime}, x_{2}{ }^{\prime}, x_{3}{ }^{\prime}\right)$ in which the $x_{3}^{\prime}$ axis coincides with the major axis of the ellipsoidal fiber were introduced (Figure 2). The local and global systems are related by the transformation: $\mathbf{x}=\boldsymbol{\beta} \mathbf{x}^{\prime}$, where $\boldsymbol{\beta}$ is the transformation matrix, whose components are functions of the angles $\theta$ and $\varphi$.

The distribution of the possible fiber orientation in the representative volume can be defined by a statistical density function $\rho(\theta, \varphi)$. In this study, a reduced density function $\rho(\theta)$ was considered for simplicity, which corresponds to a 2D case or a 3D axis symmetrical distribution.

\subsection{Linear thermomechanical constitutive relation}

The composite material was subjected to either a macroscopically uniform stress $\bar{\sigma}$ or strain $\bar{\varepsilon}$ combined with a uniform temperature change $\Delta T$, same as for the representative volume. The subscript $r$ denotes the material phase; namely $r=m$ indicates the matrix, $r=1,2 \ldots$

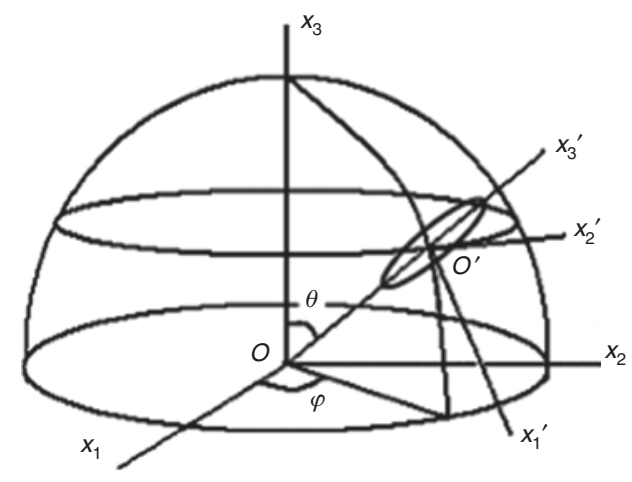

Figure 2: Schematic illustration of the global and local coordinate systems. 
$n$ indicates the $r^{\text {th }}$ fiber-reinforced phase. The stress and strain relations of each phase can be written in the following form [9]:

$$
\varepsilon_{r}=\mathbf{M}_{r}: \sigma_{r}+\mathbf{m}_{r} \Delta T, \sigma_{r}=\mathbf{E}_{r}: \varepsilon_{r}+\mathbf{e}_{r} \Delta T
$$

where $\mathbf{e}_{r}=-\mathbf{E}_{r}: \mathbf{m}_{r}, \mathbf{E}_{r}: \mathbf{M}_{r}=\mathbf{I}$, and $\mathbf{I}$ is the fourth-order unit tensor. $\boldsymbol{\sigma}_{r}$ and $\boldsymbol{\varepsilon}_{r}$ are the stress and strain tensors. $\mathbf{e}_{r}$ and $\mathbf{m}_{\mathrm{r}}$ are the unit thermal stress and strain tensors. $\mathbf{E}_{r}$ and $\mathbf{M}_{r}$ are the stiffness and compliance tensors. In the local coordinate system, $\mathbf{E}_{r}, \mathbf{M}_{r}, \mathbf{e}_{r}$ and $\mathbf{m}_{r}$ are the same for any fiber-reinforced phase.

According to the Eshelby solution of an ellipsoidal inclusion, the Mori-Tanaka average method, and the selfconsistent method, the strain and stress tensors of each phase in the linear elastic range can be determined as follows [9]:

$\boldsymbol{\varepsilon}_{r}=\mathbf{A}_{r}: \overline{\boldsymbol{\varepsilon}}+\mathbf{a}_{r} \Delta T, \sigma_{r}=\mathbf{B}_{r}: \overline{\boldsymbol{\sigma}}+\mathbf{b}_{r} \Delta T, r=\mathrm{m}$ or $1,2, \ldots n$

where $\mathbf{A}_{r}$ and $\mathbf{B}_{r}$ are the mechanical strain and stress concentration tensors of each phase, and $\mathbf{a}_{r}$ and $\mathbf{b}_{r}$ are the corresponding thermal strain and stress concentration tensors. Their expression corresponding to the $r$ th fiberreinforced phase can be obtained as follows [10-12]:

$\mathbf{A}_{r}=\left[\mathbf{I}+c_{\mathrm{m}} \mathbf{S}: \mathbf{M}_{\mathrm{m}}:\left(\mathbf{E}_{r}-\mathbf{E}_{\mathrm{m}}\right)\right]^{-1}, \mathbf{a}_{r}=\left(\mathbf{E}_{r}+\mathbf{E}^{*}\right)^{-1}:\left(\mathbf{e}_{\mathrm{m}}-\mathbf{e}_{r}\right)$

$\mathbf{B}_{r}=\left[\mathbf{I}+c_{\mathrm{m}} \mathbf{E}_{\mathrm{m}}:(\mathbf{I}-\mathbf{S}):\left(\mathbf{M}_{r}-\mathbf{M}_{\mathrm{m}}\right)\right]^{-1}, \mathbf{b}_{r}=\left(\mathbf{M}_{r}+\mathbf{M}^{*}\right)^{-1}:\left(\mathbf{m}_{\mathrm{m}}-\mathbf{m}_{r}\right)$

where $c_{r}$ denotes the volume fraction of the corresponding phase. The numerical method to calculate the Eshelby tensor $\mathbf{S}$ for a general anisotropic matrix and the ellipsoid fibers with any aspect ratio was developed by Gavazzi and Lagoudas [13]. $\mathbf{U}$ is the dual tensor of $\mathbf{S}$, and it can be calculated through Hill's equation [10] as follows: S: $\mathbf{M}_{\mathrm{m}}=\mathbf{M}_{\mathrm{m}}$ : (I-U). $\mathbf{E}^{*}$ and $\mathbf{M}^{*}$ are elastic constraint tensors whose expressions are $\mathbf{E}^{*}=\mathbf{E}_{\mathrm{m}}:\left(\mathbf{S}^{-1}-\mathbf{I}\right)$ and $\mathbf{M}^{*}=\left(\mathbf{E}^{*}\right)^{-1}=\left(\mathbf{S}^{-1}-\mathbf{I}\right)^{-1}: \mathbf{M}_{\mathrm{m}}$.

In the global coordinate system, $\mathbf{A}_{r}, \mathbf{B}_{r}, \mathbf{a}_{r}$, and $\mathbf{b}_{r}$ are dependent on the shape and orientation of the fiber-reinforced phase. After using the reduced distribution function $\rho(\theta)$, the volumetric average of concentration tensors over all the short fiber-reinforced phases $\overline{\mathbf{A}}_{\mathrm{f}}, \overline{\mathbf{B}}_{\mathrm{f}}, \overline{\mathbf{a}}_{\mathrm{f}}$, and $\overline{\mathbf{b}}_{\mathrm{f}}$ had been defined in the global coordinate system. The subscript $r=\mathrm{f}$ corresponds the fiber for the average value of all the reinforcement phases. The expression for $\overline{\mathbf{A}}_{\mathrm{f}}$ as an example is

$$
\overline{\mathbf{A}}_{\mathrm{f}}=\frac{1}{V_{\Omega}} \int_{\Omega} \mathbf{A} d V=\frac{\int_{0}^{2 \pi} \int_{0}^{2 \pi} \int_{0}^{\pi / 2}\langle\mathbf{A}\rangle \rho(\theta) \sin \theta d \theta d \varphi d \phi}{\int_{0}^{2 \pi} \int_{0}^{2 \pi} \int_{0}^{\pi / 2} \rho(\theta) \sin \theta d \theta d \varphi d \phi}
$$

where $\mathrm{V}_{\Omega}$ is the volume of all fibers and $<\mathbf{A}>$ is the volumetric average of concentration tensor $\mathbf{A}$ over a single fiber.

Because the macroscopic strain and stress are equal to the volumetric average strain and stress, then the relations between the average concentration tensors of all fibers $\overline{\mathbf{A}}_{\mathrm{f}}, \overline{\mathbf{B}}_{\mathrm{f}}, \overline{\mathbf{a}}_{\mathrm{f}}$, and $\overline{\mathbf{b}}_{\mathrm{f}}$ and the concentration tensors of the matrix $\mathbf{A}_{\mathrm{m}}, \mathbf{B}_{\mathrm{m}}, \mathbf{a}_{\mathrm{m}}$, and $\mathbf{b}_{\mathrm{m}}$ are expressed as follows [9, 11]:

$$
\begin{aligned}
& c_{\mathrm{f}} \overline{\mathbf{A}}_{\mathrm{f}}+c_{\mathrm{m}} \mathbf{A}_{\mathrm{m}}=\mathbf{I}, c_{\mathrm{f}} \overline{\mathbf{a}}_{\mathrm{f}}+c_{\mathrm{m}} \mathbf{a}_{\mathrm{m}}=\mathbf{0} \\
& c_{\mathrm{f}} \overline{\mathbf{B}}_{\mathrm{f}}+c_{\mathrm{m}} \mathbf{B}_{\mathrm{m}}=\mathbf{I}, c_{\mathrm{f}} \overline{\mathbf{b}}_{\mathrm{f}}+c_{\mathrm{m}} \mathbf{b}_{\mathrm{m}}=\mathbf{0}
\end{aligned}
$$

\subsection{Nonlinear behavior of the matrix material}

It is assumed that the fiber phase remains linearly elastic and the stress-strain relation of the matrix decides if the overall response of the composite is linear or not. In the case of ductile matrix material, the constitutive relation of composites can be extended and expressed in increment equations.

The instantaneous mechanical and thermal concentration tensors of each phase are denoted by $\hat{\mathbf{A}}_{r}, \hat{\mathbf{B}}_{r}, \hat{\mathbf{a}}_{r}$, and $\hat{\mathbf{b}}_{r}$. The Eshelby solution of an ellipsoidal inclusion is applicable. The invariant material property tensors in the elastic stage are updated considering the instantaneous material properties after each loading step in the plastic stage. When the loading parameter such as displacement or force is changed in infinitesimal time $d t$, it induces stress increment $d \overline{\boldsymbol{\sigma}}=(\partial \overline{\boldsymbol{\sigma}}(t) / \partial t) d t$. The strain and stress increments of each phase are obtained as follows:

$$
d \boldsymbol{\varepsilon}_{r}=\hat{\mathbf{A}}_{r}: d \overline{\boldsymbol{\varepsilon}}+\hat{\mathbf{a}}_{r} d T, d \sigma_{r}=\hat{\mathbf{B}}_{r}: d \overline{\boldsymbol{\sigma}}+\hat{\mathbf{b}}_{r} d T, r=\mathrm{m} \text { or } 1,2, \ldots n
$$

In the case of a metal matrix, the elastic-plastic state of a composite depends on the state of the matrix. When the matrix reaches the plastic range, the macroscopic response of the composite will be completely anisotropic, and local fields of the matrix and all fiber-reinforced phases are not only nonuniform but also depend on the loading history, that is,

$\varepsilon_{r}(\mathbf{x}, t)=\varepsilon_{r}[\mathbf{x}, \bar{\varepsilon}(t)], \sigma_{r}(\mathbf{x}, t)=\sigma_{r}[\mathbf{x}, \overline{\boldsymbol{\varepsilon}}(t)], r=\mathrm{m}$ or $1,2, \ldots n$

where $\mathbf{x}$ denotes the material spatial position. In addition, when strain control is changed to stress control, $\overline{\boldsymbol{\varepsilon}}(t)$ is replaced by $\overline{\boldsymbol{\sigma}}(t)$.

The instantaneous mechanical and thermal concentration tensors of fiber-reinforced phases are also dependent on the spatial position and loading history. The macrodependence implicitly indicates reliance on the specific microgeometry of material points, which could be 
expressed by the instantaneous stress and internal state variables of the corresponding material spatial position in the material, $\boldsymbol{\sigma}(\mathbf{x})$ and $\alpha(\mathbf{x})$ according to the internal state variable theory [14]. The average instantaneous concentration tensors of all fiber-reinforced phases in the plastic stage, $\hat{\hat{\mathbf{A}}}_{\mathrm{f}}, \hat{\mathbf{B}}_{\mathrm{f}}, \overline{\hat{\mathbf{a}}}_{\mathrm{f}}$ and $\hat{\mathbf{b}}_{\mathrm{f}}$, are assumed to be the function of the average instantaneous stress and internal state variables of the matrix, $\overline{\boldsymbol{\sigma}}_{\mathrm{m}}=\left\langle\boldsymbol{\sigma}_{\mathrm{m}}(\mathbf{x})\right\rangle$, and $\bar{\alpha}_{\mathrm{m}}=\left\langle\alpha_{\mathrm{m}}(\mathbf{x})\right\rangle$. Equation (6) becomes

$$
\begin{aligned}
& d \overline{\boldsymbol{\varepsilon}}_{\mathrm{f}}=\overline{\hat{\mathbf{A}}}_{\mathrm{f}}\left(\overline{\boldsymbol{\sigma}}_{\mathrm{m}}, \bar{\alpha}_{\mathrm{m}}\right): d \overline{\boldsymbol{\varepsilon}}+\overline{\hat{\mathbf{a}}}_{\mathrm{f}}\left(\overline{\boldsymbol{\sigma}}_{\mathrm{m}}, \bar{\alpha}_{\mathrm{m}}\right) d T \\
& d \overline{\boldsymbol{\sigma}}_{\mathrm{f}}=\hat{\hat{\mathbf{B}}}_{\mathrm{f}}\left(\overline{\boldsymbol{\sigma}}_{\mathrm{m}}, \bar{\alpha}_{\mathrm{m}}\right): d \overline{\boldsymbol{\sigma}}+\hat{\hat{\mathbf{b}}}_{\mathrm{f}}\left(\overline{\boldsymbol{\sigma}}_{\mathrm{m}}, \bar{\alpha}_{\mathrm{m}}\right) d T
\end{aligned}
$$

The expression for the average strain and stress increments of the matrix phase is similar, and the relationship between the instantaneous volume average concentration tensors of fibers and the matrix is similar to Equation (5).

It can be inferred that, in the plastic range, the average instantaneous response of all the fiber-reinforced phases is additionally dependent on the average instantaneous response of the metal matrix. The validity of this model was tested by comparing the analysis results to the available numerical and experimental results.

\subsection{Cyclic hardening effect of metal matrix}

Because the properties of a metal matrix composite in the plastic range are mainly dependent on the properties of the matrix, then attention is paid to the cyclic thermoelastic-plastic constitutive relationship of the matrix. For a time-independent metal-matrix material, the strain increment can be decomposed into a mechanical part and a thermal part:

$$
d \boldsymbol{\varepsilon}=d \boldsymbol{\varepsilon}_{\mathrm{e}}+d \boldsymbol{\varepsilon}_{\mathrm{p}}+d \boldsymbol{\varepsilon}_{\mathrm{T}}=d \boldsymbol{\varepsilon}_{\mathrm{ep}}+d \boldsymbol{\varepsilon}_{\mathrm{T}}=\mathbf{M}_{\mathrm{ep}}: d \boldsymbol{\sigma}+\mathbf{m} d T
$$

Where, $\mathbf{M}_{\mathrm{ep}}$ is the fourth-order instantaneous effective compliance tensor of the material.

It is a well established fact that most metallic materials exhibit the Bauschinger effect, which can be described by kinematic hardening. The formulation of the Bauschinger effect is based on introducing the so-called back-stress, $\mathbf{b}$, to compute the stresses and strains after plastic flow. The backstress describes the shift of the center of the yield surface.

The linear combined hardening model [15] was selected to describe the cyclic work-hardening behavior of the matrix materials. According to the $\mathrm{J}_{2}$-flow theory [16], the yield condition can be expressed by

$$
f\left(\boldsymbol{\sigma}, \sigma_{s}, \mathbf{b}\right)=\frac{1}{2}\left(\boldsymbol{\sigma}^{\prime}-\mathbf{b}^{\prime}\right):\left(\boldsymbol{\sigma}^{\prime}-\mathbf{b}^{\prime}\right)-\frac{1}{3} \sigma_{s}^{2}=0
$$

Where, $\sigma_{\mathrm{s}}$ denotes the instantaneous radius of the yield surface and $\boldsymbol{\sigma}^{\prime}$ and $\mathbf{b}^{\prime}$ are the deviatoric tensors of $\boldsymbol{\sigma}$ and $\mathbf{b}$.

Defining the cumulative plastic strain $\bar{\varepsilon}_{\mathrm{p}}$, $d \bar{\varepsilon}_{\mathrm{p}}=\sqrt{\frac{2}{3} d \varepsilon_{\mathrm{p}}: d \varepsilon_{\mathrm{p}}}, \sigma_{\mathrm{s}}$ is obtained as follows:

$$
\sigma_{\mathrm{s}}=(1-w) \sigma_{\mathrm{s}, 0}+w Y\left(\bar{\varepsilon}_{\mathrm{p}}\right)
$$

Where $\sigma_{\mathrm{s}, 0}$ is the initial yield stress, $w$ is the isotropic hardening coefficient, and $Y\left(\bar{\varepsilon}_{\mathrm{p}}\right)$ is the result in terms of the isotropic hardening model. It should be noted that $\sigma_{\mathrm{s}}$ and $Y\left(\bar{\varepsilon}_{\mathrm{p}}\right)$ are functions of $\bar{\varepsilon}_{\mathrm{p}}$.

In the case of uniaxial cyclic loading, $\bar{\varepsilon}_{\mathrm{p}}=\varepsilon_{\mathrm{p}}$, and the constitutive curve of the material is shown in Figure 3. Each monotonic loading branch is numbered as $k, k=1$, $2,3 \ldots n-1, n$. When the material reaches plasticity, for example on the $n$th loading branch, $Y\left(\bar{\varepsilon}_{\mathrm{p}}\right)$ is updated through the hardening coefficient $H$ and the hardening exponent $X$ of the material.

$$
Y_{n}\left(\bar{\varepsilon}_{\mathrm{p}}\right)=\left|\sigma_{n}\right|=\left|\sigma_{n-1}+(-1)^{n+1}\left(2 \sigma_{\mathrm{s}, n-1}+H\left|\varepsilon_{\mathrm{p}, n}-\varepsilon_{\mathrm{p}, n-1}\right|^{X}\right)\right|
$$

The material plastic modulus $E_{\mathrm{ep}}$, which is a function of $\bar{\varepsilon}_{\mathrm{p}}$, is defined under unidirectional loading as $E_{\mathrm{ep}}=d \sigma / d \varepsilon_{\mathrm{p}}$. Therefore, the expression of the instantaneous effective compliance tensor of a material could be written as

$$
\mathbf{M}_{\mathrm{ep}}=\mathbf{M}+\frac{9}{4 E_{\mathrm{ep}} \sigma_{\mathrm{s}}^{2}}\left(\boldsymbol{\sigma}^{\prime}-\mathbf{b}^{\prime}\right)\left(\boldsymbol{\sigma}^{\prime}-\mathbf{b}^{\prime}\right)
$$

To obtain an expression to determine the back-stress deviatoric tensor $\mathbf{b}^{\prime}$ during the cyclic loading process, the increment of $\mathbf{b}^{\prime}$ can be written as

$$
d \mathbf{b}^{\prime}=\sqrt{\frac{2}{3}}\left[d Y\left(\bar{\varepsilon}_{\mathrm{p}}\right)-d \sigma_{\mathrm{s}}\right] \mathbf{n}
$$

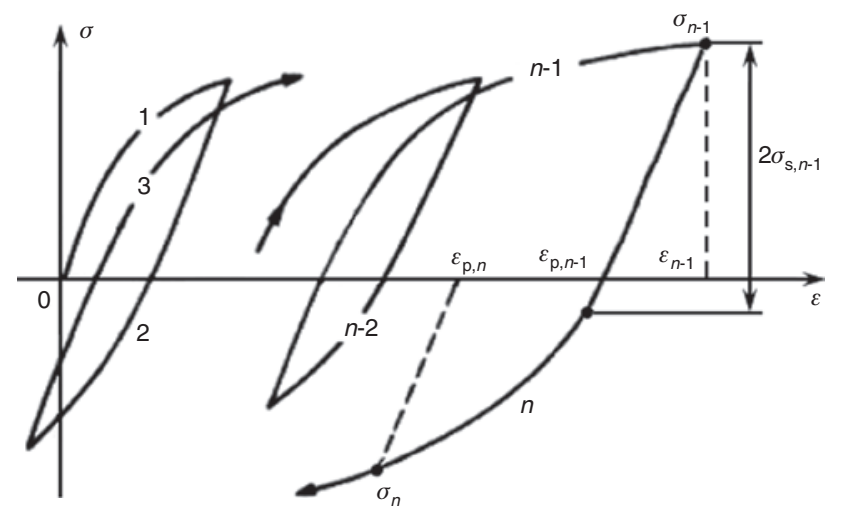

Figure 3: Constitutive curve of the material under uniaxial cyclic loading. 
Where $\mathbf{n}$ is the unit tensor normal to the material yield surface, and Eq. (14) can be solved to obtain

$$
\mathbf{b}^{\prime}=\mathbf{b}_{0}^{\prime}+(1-w) H\left(\Delta \bar{\varepsilon}_{\mathrm{p}}\right)^{X} \frac{\sigma^{\prime}}{\sigma_{\text {eq }}}
$$

Where, $\mathbf{b}_{0}^{\prime}$ is the initial back-stress deviatoric tensor at the beginning of each monotonic loading branch and the equivalent (Von-Mises) stress is $\sigma_{\text {eq }}=\sqrt{\frac{3}{2} \sigma^{\prime}: \sigma^{\prime}}$.

\section{Numerical simulation}

To express tensors in FORTRAN program, they were transformed into the form of matrices. It is assumed that the uniaxial cyclic loading is strain controlled for both mechanical and thermal loading.

Because there are unknown quantities in both the average stress and strain increments, the global constitutive relations of the representative volume are simplified:

$$
\begin{aligned}
& \Delta \overline{\boldsymbol{\varepsilon}}=c_{\mathrm{f}} \Delta \boldsymbol{\varepsilon}_{\mathrm{f}}+c_{\mathrm{m}} \Delta \boldsymbol{\varepsilon}_{\mathrm{m}} \\
& =c_{\mathrm{f}}\left(\mathbf{M}_{\mathrm{f}}: \Delta \boldsymbol{\sigma}_{\mathrm{f}}+\mathbf{m}_{\mathrm{f}} \Delta T\right)+c_{\mathrm{m}}\left(\mathbf{M}_{\mathrm{m}}: \Delta \boldsymbol{\sigma}_{\mathrm{m}}+\mathbf{m}_{\mathrm{m}} \Delta T\right) \\
& =c_{\mathrm{f}}\left(\mathbf{M}_{\mathrm{f}}:\left(\overline{\mathbf{B}}_{\mathrm{f}}: \Delta \overline{\boldsymbol{\sigma}}+\overline{\mathbf{b}}_{\mathrm{f}} \Delta T\right)+\mathbf{m}_{\mathrm{f}} \Delta T\right) \\
& +c_{\mathrm{m}}\left(\mathbf{M}_{\mathrm{m}}:\left(\mathbf{B}_{\mathrm{m}}: \Delta \overline{\boldsymbol{\sigma}}+\mathbf{b}_{\mathrm{m}} \Delta T\right)+\mathbf{m}_{\mathrm{m}} \Delta T\right) \\
& =P \Delta \overline{\boldsymbol{\sigma}}+\mathbf{Q} \\
& P=c_{\mathrm{f}} \mathbf{M}_{\mathrm{f}}: \overline{\mathbf{B}}_{\mathrm{f}}+c_{\mathrm{m}} \mathbf{M}_{\mathrm{m}}: \mathbf{B}_{\mathrm{m}} \\
& \mathbf{Q}=c_{\mathrm{f}}\left(\mathbf{M}_{\mathrm{f}}: \overline{\mathbf{b}}_{\mathrm{f}}+\mathbf{m}_{\mathrm{f}}\right) \Delta T+c_{\mathrm{m}}\left(\mathbf{M}_{\mathrm{m}}: \mathbf{b}_{\mathrm{m}}+\mathbf{m}_{\mathrm{m}}\right) \Delta T
\end{aligned}
$$

According to Equation (16), a set of equations was formed and solved to obtain the components not specified in the matrices. The average stress and strain increments of the matrix and fiber-reinforced phases were then calculated through the previous formulas. When the matrix reached the nonlinear range, an iterative procedure was required and the average stress increment of the matrix in the $i$ th step is given by

$$
\Delta \overline{\boldsymbol{\sigma}}_{\mathrm{m}}^{i}=\overline{\mathbf{B}}_{\mathrm{m}}^{i-1}\left(\overline{\boldsymbol{\sigma}}_{\mathrm{m}}^{i-1}, \bar{\alpha}_{\mathrm{m}}^{i-1}\right): \Delta \overline{\boldsymbol{\sigma}}^{i}+\overline{\hat{\mathbf{b}}}_{\mathrm{m}}^{i-1}\left(\overline{\boldsymbol{\sigma}}_{\mathrm{m}}^{i-1}, \bar{\alpha}_{\mathrm{m}}^{i-1}\right) \Delta T, i=1,2, \ldots
$$

where $\overline{\hat{\mathbf{B}}}_{\mathrm{m}}^{i-1}$ and $\overline{\hat{\mathbf{b}}}_{\mathrm{m}}^{i-1}$ are defined above, and the initial increments could be calculated through the method used for the linearly elastic range. The iterative procedure is continued until the convergence criterion is satisfied.

$$
\frac{\left\|\Delta \overline{\boldsymbol{\sigma}}_{\mathrm{m}}^{i}-\Delta \overline{\boldsymbol{\sigma}}_{\mathrm{m}}^{i-1}\right\|}{\left\|\Delta \overline{\boldsymbol{\sigma}}_{\mathrm{m}}^{i-1}\right\|} \leq \gamma
$$

where $\gamma$ is the allowable value for error. When a new loading step is applied, the above iterative procedure is repeated.

\section{Examples of the proposed model and discussion}

Lagoudas et al. [17] have compared the results of the periodic hexagonal array (PHA) model, the self-consistent model, and the Mori-Tanaka model when a multiaxial proportional loading $\left(\sigma_{33}=2 \sigma_{13}\right)$ was applied on a composite system with aluminum matrix and unidirectional distributed silicon carbide whiskers. The whisker reinforcement had a volume fraction of $24 \%$ in the composite, and the other parameters chosen for the numerical calculations are as follows: aluminum, elastic modulus $E_{\mathrm{m}}=72 \mathrm{GPa}$, Poisson ratio $v_{\mathrm{m}}=0.33, \sigma_{\mathrm{s}, 0}=70 \mathrm{MPa}$ and $H=14.5 \mathrm{MPa}$, and $\mathrm{SiC}, E_{\mathrm{f}}=430 \mathrm{GPa}$, and $v_{\mathrm{f}}=0.25$. In Figure 4, the numerical results of the proposed model are compared to the results of the PHA, Mori-Tanaka, and self-consistent models included in Ref. [17], and a good correlation is shown.

Figure 5 shows a comparison between the published experimental results [18] and the present numerical results. The composite used in the experiment was Al-6061 alloy reinforced by $\mathrm{SiC}$ whiskers with volume fraction of $28 \%$. This experiment was conducted under strain control for mechanical strain range from -0.006 to 0.006 at $300^{\circ} \mathrm{C}$. Because some of the composite material parameters had not been included in the publication, these parameters were selected to enable using the proposed model: $E_{\mathrm{m}}=72 \mathrm{GPa}, v_{\mathrm{m}}=0.33, w=0.7 ; E_{\mathrm{f}}=430 \mathrm{GPa}, v_{\mathrm{f}}=0.2$. The curve

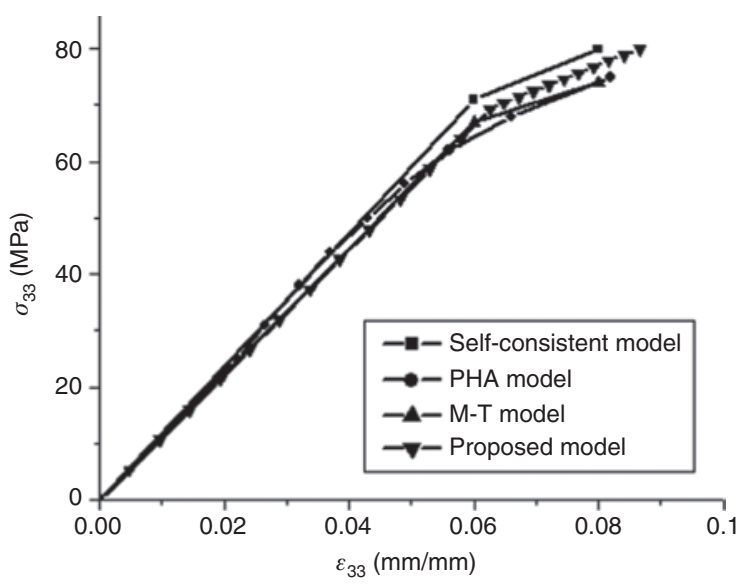

Figure 4: Comparison between the proposed model and other numerical models. 

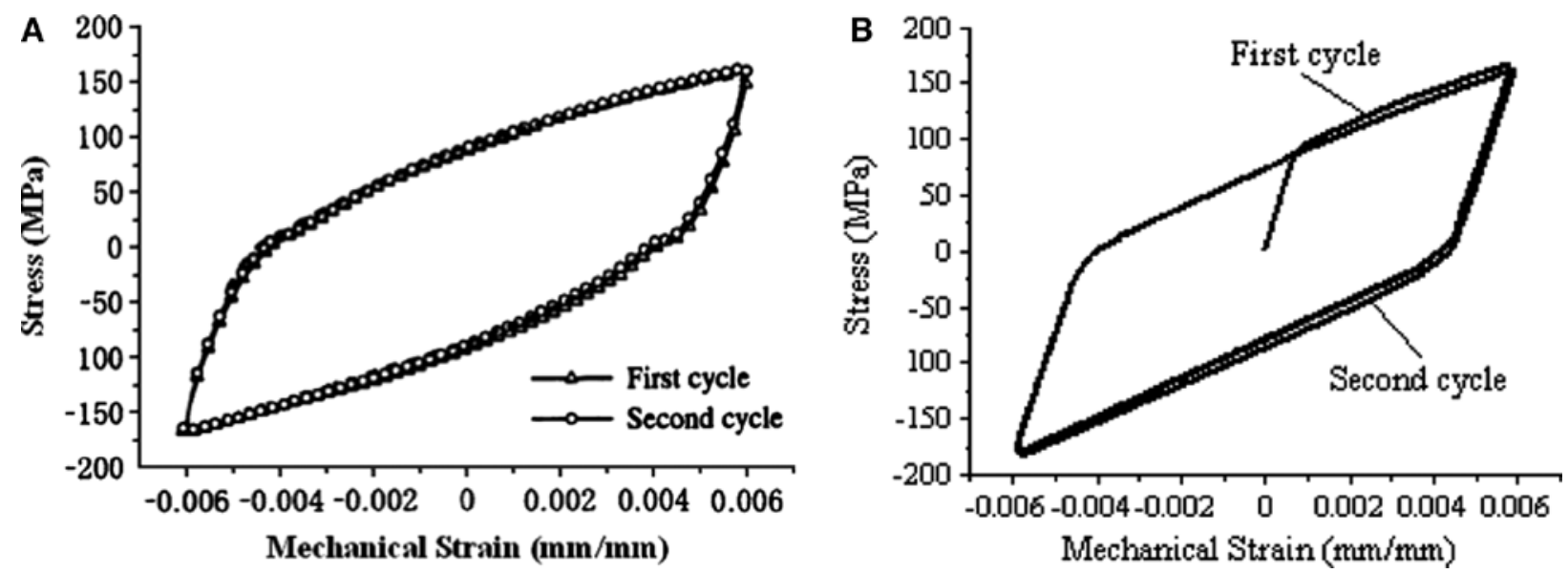

Figure 5: Comparison between calculated stress-strain responses and experimental results under isothermal loading: (A) experimental results and (B) calculated results of the proposed model.

presenting the results of this model was similar to the experimental results, except that the published results showed slight nonlinearity in the response. Better correlation to the experimental results can be achieved using the improved hardening model.

The following is a discussion of the influence of volume fraction, aspect ratio, and distribution of the spatial orientation of the fiber-reinforced phases on the stress-strain response of composites under cyclic strain control loading at room temperature.

Figure 6 illustrates the effect of volume fraction of the fiber-reinforced phases on the total stress-strain response of the composite. The composite was $\mathrm{Al}+3.5 \%$ $\mathrm{Cu}$ reinforced by $\mathrm{SiC}$ fibers, whose geometry was taken to be spherical. The aspect ratio of spherical fibers is 1:1:1 and their orientation distribution has no effect on the

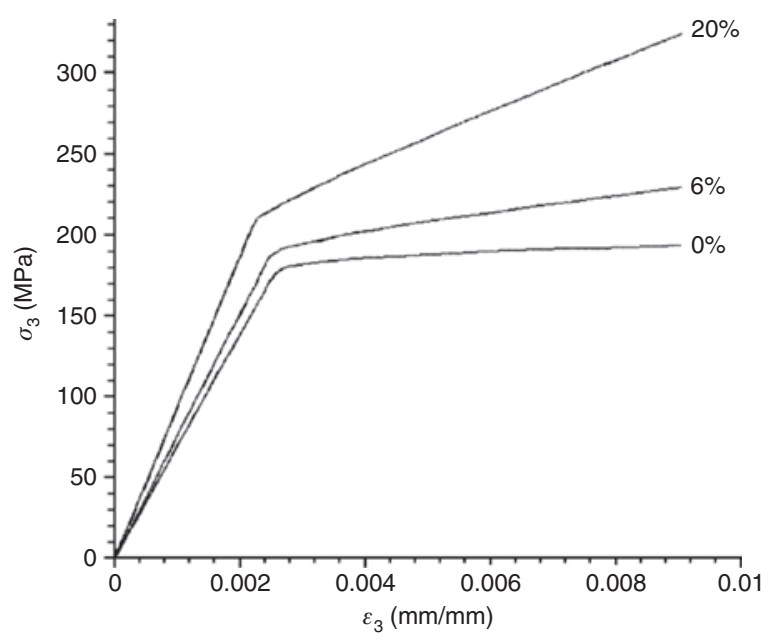

Figure 6: Stress-strain response of composite with different volume fractions of reinforcement. macroscopic response of composites. The volume fractions of the reinforcements were $0 \%, 6 \%$, and $20 \%$. Monotonic strain-controlled loading ranging from 0 to 0.009 was applied. The material properties were $E_{\mathrm{m}}=70 \mathrm{GPa}$, $v_{\mathrm{m}}=0.33, \sigma_{\mathrm{s}, 0}=175 \mathrm{MPa}, H=90 \mathrm{MPa}, X=0.25, w=0.4 ; E_{\mathrm{f}}=450$ $\mathrm{GPa}, v_{\mathrm{f}}=0.17$.

Apparently, in Figure 6, the total elastic modulus and the initial yield stress of composites increased with the fiber volume fraction. Increasing the fiber volume fraction improves the capability of the composite to support the loading, and more strain energy could be consumed under equal plastic strain. This improves the impact energy absorption characteristic, which is desired in certain civilian and military applications.

Figure 7 displays the different average stress-strain response curves of composites with different aspect ratios (1:1:1 and 1:1:5) and in different spatial orientation distributions of the short fiber-reinforced phases. The composite was Al-2124 reinforced by $\mathrm{SiC}$ fibers, with the volume fraction of $13 \%$. The material properties were $E_{\mathrm{m}}=70 \mathrm{GPa}$, $v_{\mathrm{m}}=0.33, \sigma_{\mathrm{s}, 0}=205 \mathrm{MPa}, H=220 \mathrm{MPa}, X=0.5 ; E_{\mathrm{f}}=450 \mathrm{GPa}$, $v_{\mathrm{f}}=0.17$. The short fiber orientation distributions were unidirectional, distributed in the loading direction, cosine distribution $\rho(\theta)=\cos (3 \theta / 2)$, and random distribution. The response of the unidirectional short fiber composite is shown in Figure 7A and the response of the cosine and random distributions are shown in Figure $7 \mathrm{~B}$ and $\mathrm{C}$, respectively.

Two cycles of strain-controlled loading ranging from -0.008 to 0.008 were considered, and the coefficient of isotropic hardening was defined as $w=0.2$ to accelerate the stabilization of the cyclic loops. The reinforcement effect of particles was weaker than elliptical fibers with an aspect ratio of 1:1:5 as shown in Figure 7A. Higher elastic modulus 

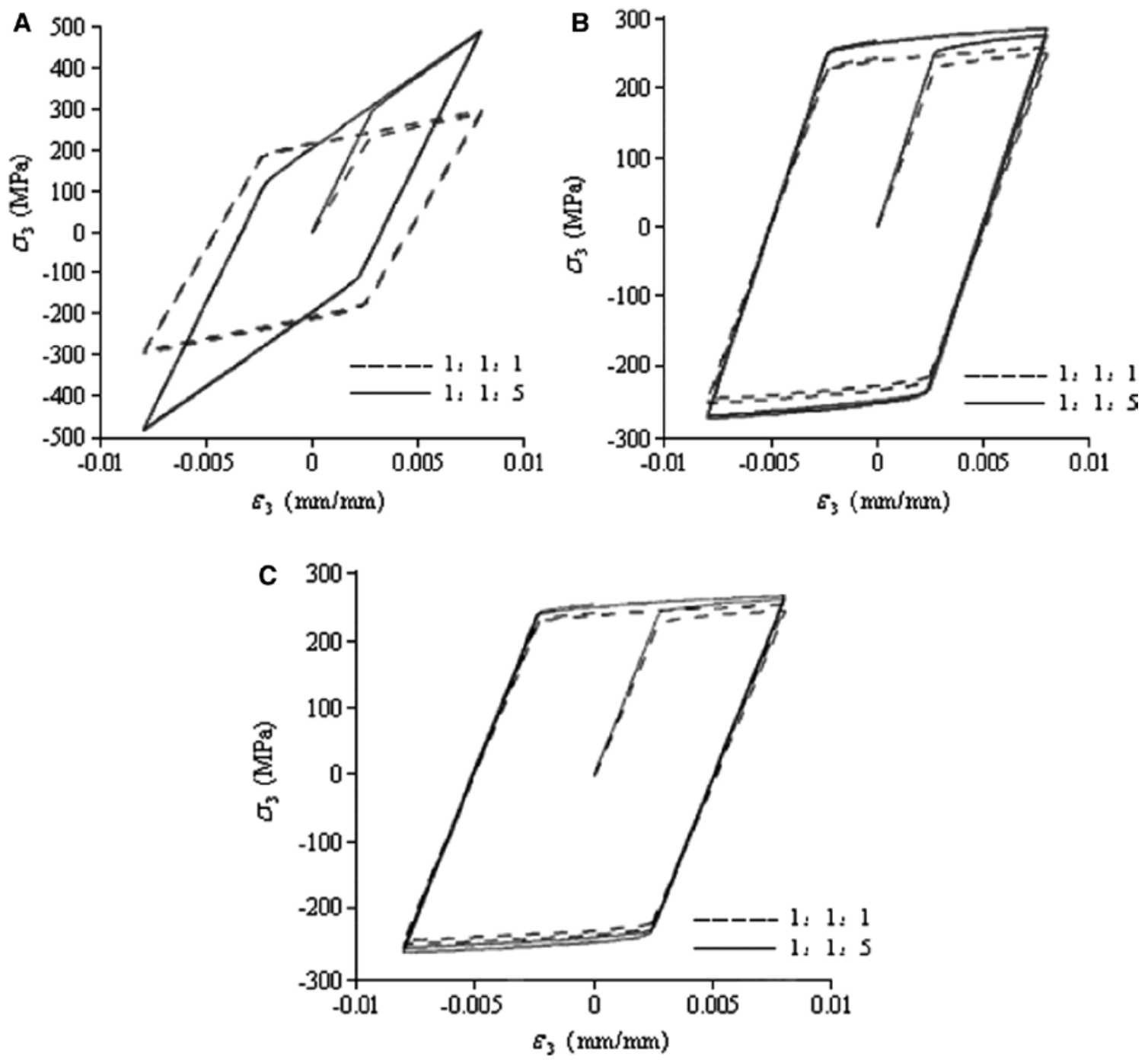

Figure 7: Stress-strain response of composite with different fiber aspect ratios: (A) unidirectional distribution, (B) cosine distribution, and (C) random distribution.

and yield strength were found with a larger aspect ratio in the loading direction. The aspect ratio of cosine-distributed short fibers did not have much influence in reinforcement as unidirectionally distributed ones because only a part of the reinforcing short fibers had their major axes along the loading direction. When the short fibers were randomly distributed, the reinforcement effect of ellipsoidal fibers to the overall response of the composite was similar to the reinforcement effect of particles, as shown in Figure 7C. Increasing the volume fraction is more efficient and easier than controlling the orientation of the fibers in short fiber composites to improve its load-carrying capacity.

Finally, we analyzed the properties of composites under coupled thermomechanical loading. Two types of thermomechanical loading, in-phase (maximum tensile strain at maximum temperature) and out-of-phase (maximum compressive strain at maximum temperature), were adopted to simulate the loading history. The total strain varied from - 0.01 to 0.01 , and the temperature range was $90^{\circ} \mathrm{C}-210^{\circ} \mathrm{C}$. The used composite was $\mathrm{Al} / \mathrm{SiC}$, and its properties were functions of temperature: $E_{\mathrm{m}}=74657[1-$ $\left.(T+273)^{3} / 933^{3}\right] \mathrm{MPa}, v_{\mathrm{m}}=0.33, m_{\mathrm{m}}=2.35 \times 10^{-5}+2.476 \times 10^{-8} T \mathrm{~K}^{-1}$; $E_{\mathrm{f}}=450 \mathrm{GPa}, v_{\mathrm{f}}=0.17$.

Figure 8 illustrates the stress-strain response for the in-phase and out-of-phase loading history. In addition, the stress-strain response for the case of isothermal loading at the average thermal loading temperature $\left(150^{\circ} \mathrm{C}\right)$ is shown in Figure 8 as reference for comparison. When the stress-strain responses for the in-phase and outof-phase loading history were compared to that of the isothermal loading, the response curve of in-phase loading was much flatter than that of isothermal loading and the corresponding stress was lower for the identical strain at the plastic stage. The out-of-phase loading demonstrated 

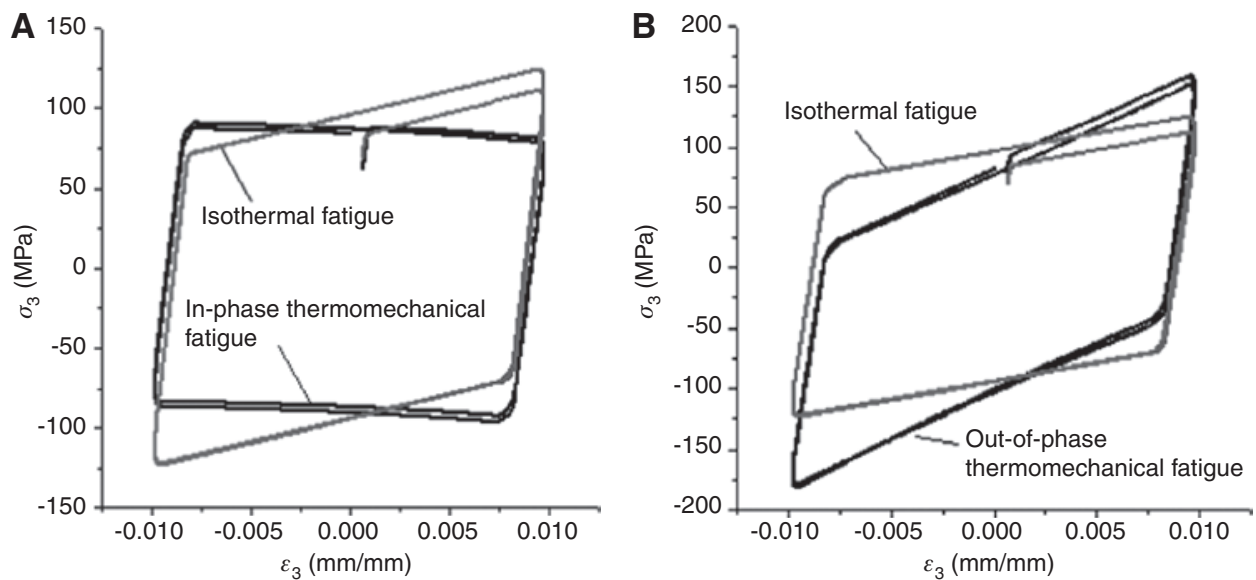

Figure 8: Stress-strain response under isothermal and thermomechanical loading: (A) in-phase and (B) out-of-phase.

the opposite phenomenon. These results can be explained by the fact that heat expansion compensates part of the mechanical strain and the thermal contraction requires less compressive mechanical strain. Furthermore, the decrease in the elastic modulus with increasing temperature also contributed to the observed results.

\section{Conclusions}

There is a good correlation between the results of the proposed model and the predicting results of the PHA model, the Mori-Tanaka model, and the self-consistent model. In addition, a good agreement was found when the results of applying the proposed model to aluminum matrix composites were compared to relevant published experimental results. The linear combined hardening model was used in the simulation and the response might be improved using a better cyclic hardening model.

The macroscopic properties of composites are dependent on the fiber's architecture and spatial orientation, especially the volume fraction of fibers. The reinforcement effect of particles is weaker than elliptical fibers, especially when unidirectional fibers are distributed in the loading direction. Increasing the fiber volume fraction improves the capability of short fiber composites to support the loading, in particular when more ellipsoidal fibers are used in the loading direction.

The numerical simulation of in-phase and out-ofphase thermomechanical loading history resulted in distinctly different stress-strain responses, which indicate the significant influence of thermal loading time history in the case of large temperature variation. The stress-strain response of the in-phase loading history was much flatter than that of isothermal loading at the average thermal loading temperature $\left(150^{\circ} \mathrm{C}\right)$.

Acknowledgments: This work was supported by the National Natural Science Foundation of China (grant number 50371042).

\section{References}

[1] Jackson KS, Wallace SFJ, Ostrem SE. Patent application publication, US 20080169380A1, 2008.

[2] Mondali M, Abedian A, Adibnazari S. Comp. Mater. Sci. 2005, 34, 140-150.

[3] Chen HF, Ponter ARS. Comp. Mater. Sci. 2005, 34, 425-441.

[4] Han W, Eckschlager A, Bohm HJ. Comput. Sci. Technol. 2001, 61, 1581-1590.

[5] Zhang F, Sun P, Li X, Zhang G. Mater. Lett. 2001, 49, 69-74.

[6] Cavalcante AAM, Pindera MJ, Khatam H. Compos. Pt. B Eng. 2012, 43, 2521-2543.

[7] Dunn ML, Taya M. J. Mater. Sci. 1994, 29, 2053-2062.

[8] Pettermann HE, Bohm HJ, Rammerstorfer FG. Compos. Pt. B Eng. 1997, 28B, 253-265.

[9] Dvorak G], Bahei-El-Din YA. J. Mech. Phys. Solids 1979, 27, 51-72.

[10] Hill R. J. Mech. Phys. Solids 1965, 13, 213-222.

[11] Hill R. J. Mech. Phys. Solids 1963, 11, 357-372.

[12] Dvorak GJ. In Metal Matrix Composites: Mechanisms and Properties, Everett RK, Arsenault RJ, Eds. Academic Press: Boston, 1991, p 1-77.

[13] Gavazzi AC, Lagoudas DC. Comput. Mech. 1990, 7, 13-19.

[14] Horstemeyer MF, Bammann DJ. Int. J. Plasticity 2010, 26, 1310-1334.

[15] Zou Y, Yun G, Zhuang Z, Kasa S, Tsunori M. Int. J. Comput. Methods Eng. Sci. Mech. 2007, 8, 181-187.

[16] Hwang KC, Huang Y, Eds. The Constitutive Relation of Solid (in Chinese). Tsinghua University Press: Beijing, 1999, p 1-27.

[17] Lagoudas DC, Gavazzi AC, Nigam H. Comput. Mech. 1991, 8, 193-203.

[18] Qian LH, Wang ZG, Toda H, Kobayashi T. Mater. Sci. Eng. A Struct. 2000, 291, 235-245. 